\title{
POUR UNE SOCIOLOGIE DE CHAIR ET DE SANG
}

Traduction de Michaël Busset et Michaël Cordey, revue par l'auteur

Loïc Wacquant

ENS Cachan | « Terrains \& travaux »

$2015 / 1 \mathrm{~N}^{\circ} 26 \mid$ pages 239 à 256

ISSN 1627-9506

Article disponible en ligne à l'adresse :

http://www.cairn.info/revue-terrains-et-travaux-2015-1-page-239.htm

\section{!Pour citer cet article :}

Loïc Wacquant, « Pour une sociologie de chair et de sang. Traduction de Michaël Busset et Michaël Cordey, revue par l'auteur », Terrains \& travaux 2015/1 ( $\left.{ }^{\circ} 26\right)$, p. 239-256.

Distribution électronique Cairn.info pour ENS Cachan.

(C) ENS Cachan. Tous droits réservés pour tous pays.

La reproduction ou représentation de cet article, notamment par photocopie, n'est autorisée que dans les limites des conditions générales d'utilisation du site ou, le cas échéant, des conditions générales de la licence souscrite par votre établissement. Toute autre reproduction ou représentation, en tout ou partie, sous quelque forme et de quelque manière que ce soit, est interdite sauf accord préalable et écrit de l'éditeur, en dehors des cas prévus par la législation en vigueur en France. Il est précisé que son stockage dans une base de données est également interdit. 


\section{Pour une sociologie de chair et de sang Traduction de Michaël Busset et Michaël Cordey, revue par l'auteur}

\section{Résumé}

Cet article élabore l'ontologie et la méthodologie sociales de la sociologie charnelle comme mode distinctif d'investigation déjouant la posture spectatrice pour saisir l'action-en-trainde-se-faire, dans le sillage des débats suscités par mon enquête par apprentissage sur la boxe comme art du corps plébéien. Je critique d'abord les notions (dualiste) d'agent, (externaliste) de structure et (mentaliste) de connaissance qui régentent les sciences sociales contemporaines et j'ébauche une conception alternative de l'animal social considéré non pas comme un simple manipulateur de symboles mais comme une créature de chair et de sang sensible, souffrante, sachant-faire, sédimentée et située. Je mets en exergue la primauté du savoir pratique incorporé qui survient des trames d'action dans lesquelles il est continuellement imbriqué, et j'examine quelles sont les modalités d'enquête aptes à déployer et à tirer profit de cette conception incarnée de l'agent. Je soutiens que l'ethnographie énactive, variante du travail de terrain par immersion fondée sur l'« effectuation du phénomène ", ouvre une voie féconde pour saisir les schèmes cognitifs, conatifs et cathectiques (habitus) qui génèrent les conduites et sous-tendent le cosmos considéré. Mais il faut de l'audace et de la ténacité sociales pour tirer parti de la "participation observante » et atteindre la compétence sociale (par opposition à la saturation empirique). Je reviens en conclusion sur le dialogue de Bourdieu avec Pascal afin de soupeser les difficultés et de souligner l'urgence de saisir l'« esprit de finesse » qui anime toute compétence mais qui disparaît des comptesrendus de la science sociale normale.

Mots clés: agent, structure, connaissance, corps, incarnation, habitus, ontologie sociale, participation observante, ethnographie énactive, Bourdieu, Pascal.

\section{I Abstract}

This article elaborates the social ontology and methodology of carnal sociology as a distinctive mode of social inquiry eschewing the spectatorial posture to grasp action-in-the-making, in the wake of debates triggered by my apprenticeship-based study of boxing as a plebeian 
bodily craft. First I critique the notions of (dualist) agent, (externalist) structure, and (mentalist) knowledge prevalent in the contemporary social sciences and sketch an alternative conception of the social animal, not just as wielder of symbols, but as sensate, suffering, skilled, sedimented, and situated creature of flesh and blood. I spotlight the primacy of embodied practical knowledge arising out of and continuously enmeshed in webs of action and consider what modes of inquiry are suited to deploying and mining this incarnate conception of the agent. I argue that enactive ethnography, the brand of immersive fieldwork based on "performing the phenomenon," is a fruitful path toward capturing the cognitive, conative, and cathectic schemata (habitus) that generate the practices and underlie the cosmos under investigation. But it takes social spunk and persistence to reap the rewards of "observant participation" and achieve social competency (as distinct from empirical saturation). In closing, I return to Bourdieu's dialogue with Pascal to consider the special difficulty and urgency of capturing the "spirit of acuteness" that animates such competency but vanishes from normal sociological accounts.

Keywords: agent, structure, knowledge, body, incarnation, habitus, social ontology, observant participation, enactive ethnography, Bourdieu, Pascal

C et article est une version amplifiée d'un texte écrit initialement en réponse à un ensemble de questions qui mont été soumises par Frank Adloff (sociologue à la Friedrich-Alexander-Universität de Nuremberg et à l'Institut universitaire européen de Florence) et ses collègues pour figurer dans un ouvrage collectif sondant la relation entre « l'incorporation et l'interprétation » dans l'analyse sociale (Adloff et al. 2014). Je me sers de ces interrogations comme d'un tremplin pour clarifier divers problèmes soulevés lors d'une série de débats autour de la théorie dispositionnelle de l'action de Bourdieu dans lesquels j'ai été impliqué durant la dernière décennie, dans le cadre de diverses conférences ou par le biais de revues, dans le sillage de mon enquête incarnée sur la boxe professionnelle en tant qu'art corporel plébéien (voir notamment le numéro spécial de la revue Qualitative Sociology à propos de Corps et âme, Auyero [2005] ainsi que le symposium sur "Habitus in Body E' Soul ", dans Theory E' Psychology, Stam [2009] et sur "Homines in Extremis », dans la revue Body $\mathcal{E}^{\circ}$ Society, 2014, ainsi que Wacquant [2008]).

Je fais d'abord la critique des notions (dualiste) d'agent, (externaliste) de structure et (mentaliste) de connaissance qui prévalent dans les sciences sociales contemporaines et j'esquisse une conception alternative de l'animal social. Je propose de considérer ce dernier non pas comme un simple créateur et manipulateur néo-kantien de symboles - à la manière d'Ernst 
Cassirer, de George Herbert Mead et de John Searle (2009 : ix), pour qui les humains sont " des êtres sociaux et politiques, conscients, rationnels, accomplissant des actes de langage et doués de libre arbitre »- mais comme une créature corporée sensible, souffrante, sachant-faire, sédimentée et située. Je mets en exergue la primauté du savoir pratique incorporé qui survient des trames d'action dans lesquelles il est continuellement imbriqué, sur lequel la maîtrise discursive vient se greffer. J'examine ensuite quelles sont les modalités d'enquête aptes à déployer et tirer parti de cette conception incarnée de l'animal humain. Je soutiens que l'ethnographie énactive, variante du travail de terrain par immersion fondée sur l'« effectuation du phénomène ", ouvre une voie féconde pour saisir les schèmes cognitifs, conatifs et cathectiques (à savoir, l'habitus) qui génèrent les conduites et sous-tendent le cosmos considéré. Mais il faut de l'audace et de la ténacité sociales pour s'enfouir dans une position appropriée de « participation observante » et en récolter tous les bénéfices. Spécifier l'ontologie et la méthodologie sociales de la sociologie charnelle me conduit à revenir sur le dialogue de Bourdieu avec Pascal afin de soupeser les difficultés et de souligner l'urgence de saisir l'« esprit de finesse » qui anime toute compétence sociale mais qui se trouve effacé des comptes-rendus de la science sociale normale.

\section{Selon vous, est-ce qu'il importe de se concentrer sur les dimensions implicites (savoir tacite, savoir-faire, sens pratique, etc.) de l'interac- tion sociale?}

C'est crucial si l'on entend surmonter trois défauts pérennes qui entravent les sciences sociales et nous empêchent de développer des comptes-rendus vibrants et colorés de la société et de l'histoire: une vision dualiste et désincarnée de l'agent, comme constitué d'un esprit actif monté sur un corps absent, inerte et imbécile; une notion de structure raplatie et négative, conçue comme un jeu de contraintes externes; et une compréhension mentaliste de la connaissance comme faite de blocs d'informations et de stocks de représentations. Ces trois conceptions se renforcent mutuellement et se lient entre elles de sorte à littéralement ôter toute vie à la vie sociale; elles nous livrent une saisie incomplète et inadéquate du social comme domaine conatif fluide mais néanmoins ordonné, au sens de patterned.

Considérons brièvement chacun de ces éléments. Les conceptions de l'agent à travers les sciences sociales sont polarisées par une opposition entre, d'un côté, l'bomo economicus, une machine computationnelle qui 
maximise l'utilité individuelle, descendu de Bentham et développé par l'économie néo-classique, et, de l'autre, l' homo culturalis, un individu manipulateur de symboles motivé par des normes morales, hérité de Kant et idolâtré par l'anthropologie culturelle, la sociologie s'étirant maladroitement entre ces deux pôles. Ces deux modèles-maîtres, «l'homme rationnel » et "l'homme plastique ", comme les a jadis qualifiés Martin Hollis (1977), sont également mutilés et mutilants. Qu'ont-ils en commun par-delà leur opposition frontale? Tous deux sont désincarnés et effacent de l'analyse la chair, le désir et la passion comme modalité de l'existence sociale. Ce sont là les ingrédients de l'action avec lesquels a bataillé William James et que la psychologie des profondeurs de Sigmund Freud ambitionnait de capturer, mais seulement à un niveau ontogénétique. Les approches en termes d'« embodiment » des sciences cognitives contemporaines, qui traversent l'intelligence artificielle, la psychologie, la neurobiologie, la linguistique et la philosophie, sont en train d'en refaire la découverte au niveau phylogénétique (Clark 1999, Lakoff and Johnson 1999, Chemero 2013, Shapiro 2014). Mais ils continuent d'être censurés, ignorés ou mis de côté par les comptes-rendus standards des sciences sociales.

Nonobstant son caractère "polythétique et polymorphe » relevé par Merton (1976), la conception prédominante de la structure sociale la place résolument à l'extérieur de l'agent, sous l'aspect d'une clôture ou d'un entonnoir, et c'est tout aussi tronqué et restrictif. Car les structures n'existent pas simplement comme des faits durkheimiens que les individus rencontrent dans leur environnement sous la forme de relations invisibles, de distributions objectives de ressources ou de systèmes de contraintes et d'opportunités qui les poussent ou les empêchent de l'extérieur. Elles sont aussi faites de mailles dynamiques de forces inscrites sur les corps et enfouies profondément dans l'organisme sous forme de grilles perceptuelles, de capacités sensorimotrices, d'inclinations émotionnelles et, bien évidemment, en tant que désir lui-même. Les structures sont des hélices internes ou des ressorts intérieurs autant qu'elles sont des conteneurs, des poutres ou des treillages extérieurs. Elles sont agiles et vivantes, et non pas inertes et immobiles.

Enfin, les sciences sociales travaillent avec une notion excessivement cérébrale et passive de la connaissance. Nous accordons la dignité de savoir aux informations propositionnelles charriées par le langage et localisées dans le cerveau. Nous négligeons les savoirs procéduraux et les connaissances pragmatiques acquises et exprimées par des actes concrets (en grec ancien, pragmaticos signifie actif, adroit en affaires privées ou publiques). Il nous faut déjouer cette conception d'en haut (top down) pour surmonter ce que 
Elizabeth Anscombe (2002: 108) nomme judicieusement une « conception de la connaissance [...] irrémédiablement contemplative » héritée de la révolution rationaliste, et rompre par là avec le concept mentaliste (ou discursiviste) de culture qui lui est associé. Nous devons reconnaitre pleinement la réalité et la puissance du savoir-faire charnel, cette prise viscérale d'en bas (bottom-up) du monde social - au double sens de compréhension intellectuelle et de manipulation habile - que nous acquérons en agissant dans et sur lui.

\section{Quelles sont les propriétés de l'acteur humain qu'il nous faut mettre en avant pour saisir cette connaissance incorporée?}

Le grand philosophe néo-kantien Ernst Cassirer (1975) caractérisait judicieusement «l'homme » d'" animal symbolique » et il voyait dans le langage, le mythe, l'art, la religion et la science les principaux systèmes symboliques inventés par les humains pour affronter leur environnement et le façonner. Mais cette caractéristique unique ne suffit pas à construire une anthropologie philosophique viable. Je lui adjoindrai cinq autres propriétés, commençant toutes commodément par la lettre $\mathrm{S}$, de sorte qu'on peut qualifier cette vision élargie de conception « Six $\mathrm{S}$ » de l'agent.

En plus d'être un manipulateur de symboles, l'animal humain est sensible, souffrant, sachant-faire, sédimenté et situé. Sensible: l'agent n'est pas seulement une personne dotée de sens, extéroceptifs, proprioceptifs et intéroceptifs; elle fait aussi sens de ce que son sensorium capture. Elle est à la fois capable de sentir et consciente de ce quelle ressent; et le corps est le médium synthétisant de cette conscience sensorielle, comme le montre le neurobiologiste Antonio Damasio dans The Feeling of What Happens (1999). Souffrant: l'agent est exposé aux menaces et aux coups du monde social et du monde naturel; il est habité de besoins, d'aspirations et de désirs qui ne trouvent pas tous satisfaction; il est constamment soumis au jugement des autres et confronté à l'avènement inévitable de la mort. En tant que telle, il vit dans l'angoisse, la détresse et la peine, et malgré tout il endure. Sachant-faire: l'agent social peut « faire la différence » (en vieux Norrois, skil, dont dérive l'anglais skilled, ${ }^{1}$ signifie originellement discerner et s'adapter) puisque, au travers de l'expérience et de l'entraînement, il acquiert des capacités d'agir et la dextérité nécessaires pour faire les choses avec compétence. Sédimenté: tous ces éléments, nos sens, la souffrance et

1. [Ndt] Dans la version originale anglaise, l'auteur utilise "skilled " que nous avons traduit par " sachantfaire " afin de respecter sa conception des « Six S " de l'agent. 
nos compétences, ne nous sont pas donnés à la naissance; ils ne sont ni génériques ni constitués dans une relation solipsiste avec soi-même. Ils sont au contraire implantés, cultivés et déployés dans le temps au fil de notre engagement dans le monde et ils sont déposés graduellement dans notre corps en tant que produit superposé de nos diverses histoires individuelles et collectives. (Merleau-Ponty [1945], s'appuyant sur Husserl, appelle le "savoir habituel du monde ", logé dans le corps propre une "science implicite ou sédimentée »). Situé: la sédimentation est façonnée par notre localisation singulière et nos pérégrinations dans l'espace physique et social, justement parce que nous sommes doublement protégés par et enfermés dans l'enveloppe physique fragile de notre organisme mortel, qui ne peut se trouver à deux endroits au même moment mais qui intègre les traces des nombreux lieux que nous avons occupés à travers le temps.

Pour finir, ces six éléments sont conjointement structurés, et ils s'entremêlent et croissent au fil du temps. Nos conceptions de l'agent, de la structure et de la connaissance doivent être radicalement temporalisées, ainsi que Bourdieu (1980 : 167-190) nous y exhortait dans le chapitre six du Sens pratique intitulé « L'action du temps ». Dès lors que nous admettons que la cognition est une activité située qui émerge d'une danse où s'enchevêtrent le corps, l'esprit, l'activité et le monde, nous pouvons nous atteler à rapatrier le savoir tacite enserré dans les pratiques culturelles et sociales et ainsi enrichir nos descriptions et approfondir les explications que nous en donnons. Réunissez ces trois ingrédients rénovés - un être incarné qui engage un savoir-faire pratique en naviguant des configurations actives et mobiles d'affects, d'actions et de pouvoirs - et vous avez les composantes de base d'une sociologie de chair et de sang capable de produire des comptes-rendus multidimensionnels et polychromes de la vie sociale qui saisissent cette vie dans son déroulement, plutôt que ces rapports torpides en noir et blanc qui remplissent aujourd'hui les revues académiques.

\section{Quelles méthodes de recherche recommandez-vous pour déceler les dimensions invisibles de l'action, de la structure et de la connais- sance?}

En principe, les quatre principales méthodes des sciences sociales - ethnographique, herméneutique (englobant la conduite d'entretiens et l'analyse textuelle), historique et statistique - peuvent toutes s'attaquer à n'importe quel objet de recherche. Mais il est clair qu'elles sont inégalement armées pour dénicher ces composants de la pratique qui ne sont pas articulés, 
symbolisés et objectivés comme tels: les catégories doxiques, les capacités phronétiques et les modes ordinaires d'être, de sentir et d'agir. Une méthode ouvre une voie royale vers la texture tacite de l'action et de la cognition sociales, celle de l'observation rapprochée s'appuyant sur l'implication pragmatique dans l'activité étudiée.

L'ethnographie, cette technique particulière de production et d'analyse de données qui repose sur l'organisme habile et sensible de l'observateur comme principal outil d'investigation, est spécialement adaptée pour ré-incarner la société en restaurant les dimensions praxéologiques de l'existence sociale. Mais, pour ce faire, il nous faut d'abord parvenir à une compréhension plus claire des vertus distinctives (et des limites corrélatives) de l'ethnographie comme enquête sociale incarnée et encastrée (embodied and embedded), adossée à la co-présence physique avec le phénomène dans son temps et son espace et, ensuite, réformer notre pratique de l'ethnographie de deux façons complémentaires bien qu'en apparence contradictoires. D'une part, nous devons arrimer l'ethnographie plus solidement à la théorie, contre les illusions épistémologiques de la « description dense " geertzienne, la naïveté philosophique de l'empirisme moral à la mode de l'École de Chicago et les séductions chic et toc du story-telling postmoderne (Wacquant 2002 : 1469-71, 1481-82; 2008 , notamment dans la partie intitulée " La chair et le texte »). D'autre part, il est indispensable de promouvoir l'implication ethnographique au long cours, intensive et même initiatique, susceptible de permettre à l'enquêteur de maîtriser à la première personne, intus et in cute, les schèmes prédiscursifs qui constituent le membre compétent, diligent et appétant dans l'univers considéré.

Pour tirer plein parti de l'ethnographie, le sociologue de terrain doit s'attacher à exploiter et à thématiser le fait que, comme tout agent social, il en vient à connaître son objet par corps; et il peut décupler sa compréhension charnelle en approfondissant son insertion sociale et symbolique dans l'univers qu'il étudie. Ce qui implique que nous pouvons et devons travailler à devenir des "observateurs vulnérables » dans notre pratique de terrain, et non pas sur le papier, en «écrivant de façon vulnérable » par l'adjonction de larges doses de "subjectivité dans l'ethnographie", comme l'a proposé Ruth Behar (1996: 16 et 6). La prescription méthodologique qui en découle est de plonger dans le flot de l'action aussi profondément que possible, plutôt que de le scruter depuis la rive; mais de plonger et nager de manière méthodique et réfléchie, et pas avec un abandon négligent qui nous ferait risquer la noyade dans le tourbillon sans fond du subjectivisme. 


\section{Dans votre ouvrage Corps et âme (Wacquant [2002], nouvelle édition augmentée en anglais 2015) et une série d'articles dérivés, vous tra- vaillez à développer ce que vous appelez une "sociologie charnelle": qu'est-ce qui la différencie d'une ethnographie sensuelle inspirée par la phénoménologie?}

Pour le dire vite, la sociologie charnelle est une sociologie non pas $d u$ corps (" of the body") en tant qu'objet socioculturel mais une sociologie à partir $d u$ corps ("from the body") en tant que source d'intelligence sociale et de sagacité sociologique. Elle s'ancre dans ce fait brut, discuté précédemment: l'agent humain est un être de chair et de sang sensible et souffrant. (La chair fait ici référence à la surface visible du corps vécu alors que le sang renvoie au système de circuits internes de la vie qui palpite dans les profondeurs du corps viscéral, ainsi que le suggère Leder (1990) dans sa relecture de Merleau-Ponty. Je rejoins ici la caractérisation de « qui nous sommes " proposée par George Lakoff et Mark Johnson dans Philosophy in the Flesh (1999 : 3-4 et passim), pour qui « l'esprit est incorporé, la pensée est principalement inconsciente » et la raison est «largement métaphorique et imaginative » ainsi qu' "émotionnellement engagée »). Elle se positionne non pas au-dessus ou à côté de l'action mais à son point de production. La sociologie charnelle cherche à éviter la posture spectatrice et à saisir l'action-en-train-de-se-faire, et non l'action-déjà-accomplie. Elle vise à détecter et à documenter le déploiement des schèmes pratiques qui façonnent la pratique: les blocs de constructions cognitifs, conatifs et affectifs de l'habitus dont l'agencement et le fonctionnement sont pleinement disponibles à l'enquête empirique (Wacquant 2014a et 2014b). Elle diverge de l'ethnographie sensuelle en tant que recherche de terrain sur les sens, dérivé d'un lignage prestigieux qui court de Simmel, Mauss et $\mathrm{Lu}-$ cien Febvre à Elias et la Lebensphilosophie d'Arnold Gehlen et de Helmut Plessner jusqu'aux courants contemporains de l'anthropologie médicale et phénoménologique, en ceci qu'elle prend les « constellations sensorielles » non pas pour objet d'étude (comme le fait Howes 2003) mais comme moyen d'investigation.

La sociologie charnelle s'applique à tout objet et peut déployer une panoplie variée de méthodes pour autant qu'elle considère l'agent social comme incarné et encastré. Par exemple, les praticiens de l'Alltagsgeschichte, de la micro-histoire et de la récente historiographie des sensibilités s'inscrivent bien souvent dans son périmètre, quand bien même ils ne le savent ou le souhaitent pas. Quand Carlo Ginsburg (1976), dans Il formaggio e i vermi, reconstruit le cosmos vécu du meunier Menocchio au XVI ${ }^{e}$ siècle, avant 
que ce dernier ne périsse sur le bûcher par suite de son hérésie suspectée; quand Alf Lüdtke (1993) traque les racines et les effets sociaux de l'Eigensinn, cette « fidélité à soi » obstinée des ouvriers allemands du premier $\mathrm{XX}^{\mathrm{e}}$ siècle qui nourrit leurs stratégies de récalcitrance à mi-chemin entre l'accommodement et la résistance au pouvoir; quand Alain Corbin (1988) cartographie les cultures sensorielles et épistémiques évolutives qui ont fait du rivage, objet traditionnel de peur révulsive, un site séducteur et un lieu de contemplation, annonçant une nouvelle posture envers la "nature ", ils font tous de la sociologie historique charnelle.

Pour ce qui concerne les objets contemporains, la meilleure méthode est celle que je qualifie maintenant d'ethnographie énactive, à savoir un travail de terrain immersif au fil duquel le chercheur joue (au sens de «acts out») ou mime le phénomène de sorte à éplucher les couches de ses propriétés invisibles et à en tester les mécanismes opératoires. J'adapte le terme énactif du philosophe Alva Noë, mon collègue de Berkeley qui, dans son livre Action in Perception (2004 : 2) argue que la perception est « une activité habile du corps de l'animal dans son ensemble », ce qui me parait une caractérisation très pertinente de l'ethnographe au travail. (Noë lui-même emprunte l'adjectif «énactif » à l'influente théorie de "l'esprit incarné » (embodied mind) de Francesco Varela, Evan Thompson et Eleanor Rosch [1991]). Le premier commandement de l'enquête incarnée est ainsi d'entrer sur le théâtre de l'action dans une capacité ordinaire et, autant que faire se peut, se placer en apprentissage des us des agents étudiés - qu'ils soient pugilistes, professeurs ou politiciens - de sorte à acquérir une appréhension viscérale de leur univers comme matériau et tremplin pour sa reconstruction analytique.

\section{Mais que faire de ces situations dans lesquelles, pour des raisons pra- tiques, légales ou morales, on ne peut pas se transformer en compo- sante du phénomène?}

Bien sûr, il n'est pas toujours facile et évident de monter et de jouer sur la scène sociale envisagée: devenir un membre actif prend généralement du temps, requiert des qualités spécifiques ou encore l'obtention de titres et certificats. Il se peut bien que vous ne possédiez pas le sang-froid nécessaire pour être policier ou la souplesse minimale pour être ballerine; vous ne pouvez pas escompter devenir juge d'application des peines à courte échéance et il est heureux qu'on ne vous autorise pas à effectuer une chirurgie du cerveau dans un hôpital d'élite à seule fin de compréhension sociologique. Mais vous pourriez bien devenir machiniste pour 
le ballet, vous inscrire comme stagiaire volontaire dans un service de probation ou encore travailler comme aide-soignant en salle d'opération. Il $\mathrm{y}$ a toujours plusieurs portes et corridors qui conduisent sur « les lieux de l'action" - "where the action is ", pour évoquer l'article classique de Goffman (1974) - et donc autant d'opportunités de faire l'expérience et d'expérimenter avec les composantes du phénomène en apprenant à tenir un rôle, aussi mineur soit-il, et à assumer la position de tel ou tel des protagonistes du drame social considéré.

Le plus souvent, les sociologues échouent à franchir la porte parce qu'il ne leur vient même pas à l'idée d'y frapper pour vérifier jusqu'où ils pourraient être invités à entrer. Un exemple: on dispose de douzaines de monographies sur les sans-abri, certaines tout à fait admirables - dans des veines différentes, je recommande le travail de Snow et Anderson (1993), Down on their Luck, le livre de Bourgois et Schoenberg (2009), Righteous Dopefiend, et l'étude de Teresa Gowan (2010), Hobos, Hustlers, and Backsliders. Aucun de leurs auteurs n'a pourtant dormi dans la rue durant de longues périodes; aucun n'a emménagé dans ces hôtels borgnes qui louent des chambres à la semaine ( $S R O$, single room occupancy hotels) aux déshérités, ou n'a été pensionnaire dans un centre d'hébergement - sans parler de circuler entre ces trois sites qui, avec les maisons d'arrêt et les hôpitaux publics, forment la trame des institutions interconnectées qui prennent en charge les personnes sans logement lorsque leur condition devient critique. (Il faut remonter jusqu'au travail collectif de Sutherland et Locke, financé par la Work Progress Administration de Roosevelt, Twenty Thousand Homeless Men (1936), pour trouver une monographie dans laquelle dormir dans les abris d'urgence est l'élément central du dispositif d'enquête). Le chercheur-type sur les sans-abri se retire de la scène à la tombée la nuit, au moment même où se pose et se résout tant bien que mal le problème pratique et existentiel autour duquel pivote cet univers: où vais-je dormir ce soir? Et pourtant il y a tant à apprendre en éprouvant de première main (ou de premier dos), nuit après nuit, les différentes techniques déployées pour se faire un « lit » de fortune convenable dans la rue et hors la rue, pour gérer les relations friables que cela implique, pour s'assurer d'une sécurité physique minimale, pour apprivoiser ses douleurs et ses émotions, s'imprégner des odeurs, altérer son sens du temps, et ainsi de suite. Autant de facettes du phénomène qui gagnent à être révélées par corps, par l'énaction même, fût-elle volontaire et temporaire dans le cas de l'enquêteur, du sansabrisme, parce qu'elles sont tissées dans la trame même de l'action urgente mais banale et qu'elles sont de ce fait devenues pour partie imperceptibles et indescriptibles par ceux qui y sont le plus aguerris. 
Linvestigation incarnée exige un zeste d'audace et beaucoup de ténacité. Outre le fait qu'elles négligent la nature incarnée de l'action, de la connaissance et de la structure, une raison majeure pour laquelle la plupart des ethnographies retombent dans la vision textuelle ou herméneutique que nourrit une posture contemplative à l'égard du monde social (interdite par définition à ceux qui pratiquent la "participation observante ») est que leurs auteurs ne persévèrent pas dans leurs efforts pour gagner accès et approfondir leur encastrement dans la scène considérée. Ils calent et battent promptement en retraite au lieu d'aller de l'avant et d'essayer tous les itinéraires possibles pour pénétrer dans l'arène de l'action. La ténacité finit pourtant bien souvent par payer: Joan Cassell (1991) s'est vue refuser l'accès à l'univers masculin du bloc opératoire et fut invitée à étudier plutôt les auxiliaires de santé féminines (ladies' auxiliary). Mais, à force d'obstination, elle a fini par observer des chirurgiens à l'œuvre dans une douzaine d'hôpitaux, deux réseaux de soins de type $\mathrm{HMO}$ et une clinique publique réalisant quelque deux cents opérations. Cassel avait prévu de conduire son enquête en 18 mois; elle a passé un total de 33 mois sur le terrain, au bout desquels elle fut autorisée à entrer sur scène et à manipuler les rétracteurs comme les internes.

De même, Gretchen Purser $(2012 ; 2015)$ a passé près de trois ans à se faire embaucher par des agences d'emploi journalier (day-labor agencies) dans les quartiers déshérités d'Oakland et de Baltimore. Ce qui l'a mise en position de produire une analyse incarnée des stratégies et des expériences sociales des travailleurs marginaux «just-in-time » piégés au carrefour du marché de l'emploi dérégulé, de l'hyperghetto dénudé et du système carcéral surdéveloppé. Quelle est la probabilité qu'une jeune femme blanche menue, plutôt gauche, végétalienne et à lunettes, se sorte de l'empoignade quotidienne que se livrent les repris de justice noirs pour les «tickets» (contrat de travail à la journée) distribués par un " atelier à viande » (body shop) et s'acquitte des boulots les plus dégradants? Et pourtant elle a enduré et elle revient de cette expérimentation sociale avec un compte-rendu incandescent de la réalité de chair et de sang de la «flexploitation » dans les bas-fonds de l'économie étasunienne.

La sociologie charnelle s'appuie sur un syllogisme et sur un défi. Le syllogisme est le suivant: s'il est vrai que le corps n'est pas seulement socialement constr-uit mais aussi un vecteur socialement constr-ucteur de connaissance, de pratique et de pouvoir, alors cette proposition s'applique au corps du sociologue en sa capacité d'enquêteur. Le défi consiste à surmonter deux millénaires de "mépris du corps ", pour parler comme Nietzsche, qui nous ont conduit à considérer l'organisme sensible comme 
un obstacle de sorte à en faire une ressource prolifique pour l'investigation sociologique. Déployer méthodiquement son corps propre en tant qu'instrument intelligent de production pratique de connaissance accélère l'acquisition des compétences sociales de base - les capacités opératoires de sentir, penser et agir à la manière d'un Nimportequi parmi les Nimportequoiqui, en retour, fournit un meilleur critère que la saturation des données pour décider du moment où votre mission est accomplie.

\section{L'« ethnographie énactive " est-elle une nouvelle manière de pratiquer le métier?}

Je serais tenté de dire, au contraire, que c'est une vieille et vénérable variante de l'ethnographie, qui nous ramène à ses origines et à son âge d'or, où l'on insistait sur le learning by doing à travers le contact intime et de longue durée avec les « indigènes »- dans sa Poétique, Aristote nous rappelle que les hommes acquièrent leurs connaissances premières par l'imitation (mimésis). L'ethnographie énactive se contente de rendre explicite puis d'intensifier les deux traits distinctifs de toute ethnographie digne de ce nom: l'enquêtrice est incarnée, elle est corps connaissant et intelligent; et elle s'est encastrée dans la structure sociale et symbolique considérée. Une autre manière de la caractériser est de dire qu'elle traite de l'habitus à la fois comme objet et comme méthode d'analyse (Wacquant 2010): l'enquêteur porte au jour et teste la pertinence des schèmes pratiques en les acquérant in actu, en se concentrant intensivement sur les techniques pédagogiques au moyen desquelles ils sont forgés, ou en disséquant les réalisations pratiques qui les mettent en œuvre. De ce point de vue, l'ethnographie énactive s'oppose aux courants de l'anthropologie contemporaine qui sont obsédés par les tropes, la " positionalité » du chercheur, l'éthique, la transparence et la multiplicité des sites (ou encore des "para-sites" nichés dans la "complexité de notre temps ", no joke) et par les déboires de la professionnalisation (Faubion et Marcus, 2009) au point d'atteindre un état de paralysie ethnographique par méta-analyse.

J'avais précédemment proposé qu'à chaque fois que les conditions pratiques l'autorisent, nous pouvons et nous devons " "go native", mais "go native" armés, et revenir " (Wacquant 2008), ce qui est une manière de corriger la vision du travail de terrain qui prévaut aujourd'hui dans la science sociale normale. Je souhaite maintenant amender cette formulation car "going native» est un slogan ambigu qui nous fait démarrer sur le mauvais pied épistémique. Qu'est-ce en effet que ce " point de vue de l'indigène » canonisé par Malinowski (1922) dans Argonauts of the 
Western Pacific, que Geertz (1974) nous enjoint d'honorer comme le point de vue de l'ethnographie? C'est le point de vue de qui et à quel moment du temps au juste? Même dans une société de petite échelle fondée sur la parenté et approchant la « solidarité mécanique » de Durkheim, telles que les îles Trobriand, il y a de la différenciation et de la hiérarchie sociales. De fait, Malinowski souligne les distinctions de rang et de pouvoir entre districts, tribus et clans totémiques. Le point de vue du chef de village n'est bien évidemment pas celui de ses manants; les perspectives et dispositions d'un veuf senior issu d'un lignage de haut rang ne sont pas celles d'une adolescente célibataire issue d'un clan totémique inférieur. Tout système d'action s'adosse à une division du travail telle qu'il existe des points de vues, pluriels, soit autant de vues prises à partir de points évolutifs dans la structure objective de l'espace social local. En outre, une lutte permanente se joue pour déterminer quelles propriétés et positions définissent l'« indigène »: qui est un membre authentique et qui ne l'est pas? La réponse à cette question est toujours en jeu dans le monde social et elle ne peut par conséquent être tranchée par un décret du sociologue. Comme je le montre dans The Prizefighter's Three Bodies (à paraître), tout point de vue singulier, tel que "le point de vue pugilistique », est toujours une construction sélective et synthétique de l'analyste qui saisit un moment dans cette dispute dynamique entre des perspectives situées, et non pas une induction empirique brute qui jaillirait telle quelle de l'observation ethnographique.

\section{Dans son plus grand livre, les Méditations Pascaliennes, Bourdieu (1997) approfondit sa théorie de la connaissance implicite à travers un dialogue voilé avec Blaise Pascal. Quel sens donner à cette auto-affilia- tion de Bourdieu avec l'auteur des Pensées (Pascal 1976)?}

Cette connexion en a surpris plus d'un car Pascal est un penseur obscur et énigmatique qui, outre ses contributions à la géométrie et à la théorie des probabilités, est plutôt connu comme apologiste du christianisme, penseur des abysses existentielles et maître-styliste en prose. Rares sont les chercheurs en sciences sociales qui l'ont rencontré dans leurs tribulations intellectuelles et Bourdieu lui-même l'a peu mentionné ouvertement dans ses écrits. Or, cette connexion opère à plusieurs niveaux qui s'emboîtent. C'est d'abord une référence prophylactique visant à écarter les fausses filiations (ainsi les accusations d'un certain maccarthysme attardé selon lesquelles Bourdieu serait un crypto-marxiste) et les mauvaises lectures (Bourdieu comme tenant d'une vision stratégique de l'action dans le moule de la « théorie du choix rationnel », dont il était un opposant rude et infatigable). C'est ensuite un 
clin d'œil ironique à la philosophie du sujet comme conscience souveraine, qui court de Descartes à Sartre en passant par Husserl (et ses Méditations cartésiennes, titre sur lequel Bourdieu « riffe»), avec laquelle le sociologue a croisé le fer pendant pratiquement quarante ans. Avec Leibniz et particulièrement Spinoza (autre inspiration majeure de Bourdieu et, comme lui, adepte ardent d'une conception moniste de l'activité humaine), Pascal représente l'aile non-cartésienne de la révolution rationaliste, que l'auteur de La Distinction entend renforcer et prolonger.

Mais il y a surtout une affinité profonde au niveau de l'anthropologie philosophique et de l'ontologie sociale: l'humain est un être souffrant, pris dans et par le monde, qui ne s'arrache à l'absurdité totale de sa condition, pris qu'il est "entre l'infinité et le néant ", que par la connaissance et l'action, même si cette connaissance ne peut jamais être fondée. Cet être et ce monde sont pétris d'antinomies, de couples hiérarchiques susceptibles de renversement du tout au tout; l'humain est à la fois « ange et bête ", soumis aux déterminismes de l'univers et pourtant capable de leur échapper par cela même qu'il peut les connaître et donc se connaître; les institutions se donnent comme fondées en nature alors qu'elles ne sont que « coutume » (« cette seconde nature qui détruit la première »); l'ordre social apparaît nécessaire alors même qu'il est foncièrement contingent. Arbitraire des hiérarchies qui dépendent du « désir capricieux des hommes » et incommensurabilité des pouvoirs («l'ordre des corps, lordre des esprits, l'ordre de la charité »), centralité du symbolique, rôle de la tromperie et de la foi (au sens de fides, confiance que les choses sont ce qu'elles paraissent être: « nier, croire et douter sont à l'homme ce que le courir est au cheval ») comme ressort de l'action et liant de la vie collective. Pascal offre à Bourdieu non pas un système - que l'auteur des Provinciales n'a jamais produit - mais des appuis et des leviers pour " affronter dans sa vérité l'énigme de la fiction et du fétichisme » (Bourdieu 1997 : 15) qui sont au fondement de la société et ainsi opérer une triple historicisation: de lêtre, du monde social qu'il fait et qui le fait, et du savoir qu'on peut en produire.

\section{Peut-on faire appel à Pascal pour approfondir notre compréhension des multiples façons dont la connaissance tacite opère dans la vie quo- tidienne, en se voilant et se dévoilant à la fois? Peut-il nous aider à développer une sociologie de l'implicite?}

«La sensibilité de l'homme aux petites choses et l'insensibilité aux plus grandes choses, marque d'un étrange renversement ", écrit Pascal (1976), et pourtant « c'est la condition des hommes ». Bourdieu enrôle le philo- 
sophe janséniste principalement pour avancer sa réflexion sur le pouvoir symbolique, ses modalités et ses effets, et pour penser la reconnaissance comme commutateur de l'existence sociale. Cela le conduit à forger la triade conceptuelle de la connaissance, de la reconnaissance et de la méconnaissance dans la vie quotidienne ainsi que dans le fonctionnement institutionnel à l'épicentre de son ontologie sociale. On peut aller plus loin et déployer le couple chamailleur "des raisons du cœur et de la raison » pour prendre au sérieux les croyances profanes et développer une microsociologie politique fusionnant rationalité et sentiments. De même, avec le concept de « divertissement » et l'idée corolaire que « ce n'est que la chasse, et non pas la prise » que les hommes recherchent dans toute activité, Pascal ouvre grandes les portes d'une sociologie de la passion comme modalité de notre rapport au monde, impliquant amour, désir et souffrance, et qui prend des formes indéfiniment diverses (philosophique, politique, pugilistique, amoureuse, etc.).

Enfin, au début de ses Pensées, Pascal introduit une distinction subtile mais lumineuse entre « l'esprit de géométrie » et « l'esprit de finesse », deux modalités de raisonnement sur lesquelles les chercheurs en sciences sociales seraient bien avisés de se pencher (voir Force [2003] pour les rudiments et Pascal [1985] pour les approfondissements). Notre esprit géométrique procède d'un petit nombre de principes pour découper le monde, déduire avec logique et conclure avec clarté; par contraste, la finesse intellectuelle se nourrit d'une multitude de principes implicites confortablement installés dans l'expérience et à cheval sur l'intuition locale et l'analogie ressentie. Le premier est abstrait et artificiel, né d'un entraînement spécialisé de l'esprit; la seconde est concrète et naturelle, qui jaillit synthétiquement du flux de la vie et, nous le savons désormais grâce aux neurosciences, enracinée dans des mouvements sensorimoteurs, donc dans l'action du corps dans et sur le monde. Pascal compare l'esprit de géométrie au regard fixe et l'esprit de finesse à la palpation de la main. Cette opposition peut nous aider à discerner la difficulté particulière des sciences sociales qu'exacerbe l'étude de l'implicite: la science normale fonctionne selon l'esprit de géométrie tandis que la vie sociale, elle, opère selon l'esprit de finesse. La tâche d'une sociologie incarnée doit dès lors être de réconcilier ces deux formes divergentes de la raison. "Nous sommes automates autant qu'esprit ", pointe Pascal, et c'est pour cette raison que nous devons nous efforcer d'éviter « deux excès: exclure la raison, n'admettre que la raison ». Ce qui nest pas un mauvais axiome de départ pour une sociologie de chair et de sang. 


\section{i u remerciements}

Je remercie Frank Adloff, Javier Auyero, Sarah Brothers, Megan Comfort, Magnus Hörnqvist, Nazli Ökten et John Searle pour leurs réactions promptes et leurs suggestions sagaces à propos de cet article et de ses arguments.

\section{1 u références}

Adloff F., Gerund K., Kaldewey D. (dir.), 2014. Revealing Tacit Knowledge: Embodiment and Explication, Berlin, Transcript Verlag.

Anscombe G. E. M., 1957. Intention, Cambridge, Harvard University Press [tr. fr. 2002, LIntention, Paris, Gallimard].

Auyero J. (dir.), 2005. Body and Soul (numéro spécial), Qualitative Sociology, 28(2), 141-211.

Behar R., 1996. The Vulnerable Observer: Anthropology that Breaks your Heart, Boston, Beacon Press.

Bourdieu P., 1980. Le Sens pratique, Paris, Éditions de Minuit.

Bourdieu P., 1997. Méditations pascaliennes, Paris, Éditions du Seuil.

Bourgois P., Schonberg J., 2009. Righteous Dopefiend, Berkeley, University of California Press.

Cassell J., 1991. Expected Miracles: Surgeons at Work, Philadelphia, Temple University Press.

Cassirer E., 1944. An Essay on Man, New Haven, Yale University Press [tr. fr. 1975, Essai sur l'homme, Paris, Éditions de Minuit].

Chemero A., 2013. Radical Embodied Cognitive Science, Review of General Psychology, 17(2), 145-150.

Clark A., 1999. "An Embodied Cognitive Science?”, Trends in Cognitive Sciences, 3(9), 345-351.

Corbin A., 1988. Le Territoire du vide. L'Occident et le désir du rivage, 1750-1840, Paris, Aubier.

Damasio A. R., 1999. The Feeling of what Happens: Body and Emotion in the Making of Consciousness, New York, Harcourt Brace [tr. fr. 1999, Le sentiment même de soi. Corps, émotions, conscience, Paris, Odile Jacob].

Faubion J. D., Marcus G. E. (dir.), 2009. Fieldwork is Not what it Used to be: Learning Anthropology's Method in a Time of Transition, Ithaca, Cornell University Press.

Force P., 2003. Géométrie, finesse, et premiers principes chez Pascal, Romance Quarterly, 50(2), 121-130. 
Geertz C., 1974. "From the Native's Point of View": On the Nature of Anthropological Understanding, Bulletin of the American Academy of Arts E Sciences, 26-45 (réédition dans 1983, Local Knowledge: Further Essays in the Interpretation of Cultures, New York, Basic Books, pp. 55-71) [tr. fr. 1986, "Du point de vue de l'indigène ». Sur la nature de la compréhension anthropologique, in Savoir local, savoir global. Les lieux du savoir, Paris, PUF, pp. 71-90].

Ginzburg C., 1976. Il formaggio e i vermi, Turin, Einaudi [tr. fr. 1993, Le Fromage et les vers, Paris, Aubier-Montaigne.]

Goffman E., 1967. Interaction Ritual: Essays on Face-to-Face Behavior, New York, Anchor [tr. fr. 1974, Les rites d'interaction, Paris, Éditions de Minuit.] Gowan T., 2010. Hobos, Hustlers, and Backsliders: Homeless in San Francisco, Minneapolis, University of Minnesota Press.

Howes D., 2003. Sensual Relations: Engaging the Senses in Culture and Social Theory, Ann Arbor, University of Michigan Press.

Hollis M., 1977. Models of Man: Philosophical Thoughts on Social Action, New York, Cambridge University Press.

Lakoff G., Johnson M., 1999. Philosophy in the Flesh: The Embodied Mind and its Challenge to Western Thought, New York, Basic Books.

Leder D., 1990. Flesh and Blood: A Proposed Supplement to MerleauPonty, Human Studies, 13(3), 209-219.

Lüdtke A., 1993. Eigen-Sinn: Fabrikalltag, Arbeitererfabrungen und Politik vom Kaiserreich bis in den Faschismus, Hambourg, Ergebnisse.

Malinowski B., 1922. Argonauts of the Western Pacific: An Account of Native Enterprise and Adventure in the Archipelagoes of Melanesian New Guinea, Londres, Routledge [tr. fr. 1963, Les Argonautes du Pacifique Occidental, Paris, Gallimard]. Merleau-Ponty M., 1945. Phénoménologie de la perception, Paris, Gallimard. Merton R., 1976. Structural Analysis in Sociology, in P. Blau (dir.), Approaches to the Study of Social Structure, Londres, Open Books, 21-52.

Noë A., 2004. Action in Perception, Cambridge, MIT Press.

Pascal B., 1985 [1657]. De l'Esprit géométrique, Paris, Garnier-Flammarion. Pascal B., 1976 [1670]. Pensées, Paris, Garnier-Flammarion.

Purser G., 2012. "Still Doin'Time": Clamoring for Work in the Day Labor Industry, WorkingUSA: The Journal of Labor \& Society, 15(3), 397-415. Purser G., 2015. Labor on Demand: Dispatching the Urban Poor, Berkeley, University of California Press.

Searle J., 2009. Making the Social World. The Structure of Human Civilization, New York, Oxford University Press.

Shapiro L., (dir.), 2014. The Routledge Handbook of Embodied Cognition, Londres, Routledge. 
Snow D.A., Anderson L., 1993. Down on their Luck: A Study of Homeless Street People, Berkeley, University of California Press.

Stam H. J. (dir.), 2009. Special Section on Habitus in the Work of Loïc Wacquant, Theory \& Psychology, 19(6), 707-777.

Sutherland E., Locke H. J., 1936. Twenty Thousand Homeless Men: A Study of Unemployed Men in the Chicago Shelter, Chicago, Lippincott (rééd. 1971, New York, Arno Press).

Varela F., Thompson E., Rosch E., 1991. The Embodied Mind: Cognitive Science and Human Experience, Cambridge, MIT press [tr. fr. 1999, L'inscription corporelle de l'esprit. Sciences cognitives et expérience humaine, Paris, Seuil]. Wacquant L., 2002 (2000). Corps et âme. Carnets ethnographiques d'un apprenti-boxeur, Marseille, Agone (version augmentée : Body and Soul: Notebooks of an Apprentice Boxer, New York, Oxford University Press, 2014).

Wacquant L., 2002. Scrutinizing the Street: Poverty, Morality, and the Pitfalls of Urban Ethnography, American Journal of Sociology, 107(6), 1468-1532.

Wacquant L., 2008. Le corps, le ghetto et l'État pénal, Labyrinthe, 31, 71-91.

Wacquant L., 2010. L'habitus comme objet et méthode, Actes de la recherche en sciences sociales, 174, 109-121 ("Habitus as Topic and Tool: Reflections on Becoming a Prizefighter", Qualitative Research in Psychology, 8(1), 81-92).

Wacquant L., 2014a. Homines in extremis: What Fighting Scholars Teach us about Habitus, Body E' Society, 20(2), 3-17.

Wacquant L., 2014b. Putting Habitus in its Place: Response to the Symposium (Symposium sur Homines in extremis, avec Nick Crossley, Greg Downey, Hélène Mialet, and Élise Paradis), Body E' Society 20(2), 118-139. Wacquant L., à paraitre. The Prizefighter's Three Bodies: Steps to a Carnal Sociology, New York, Oxford University Press.

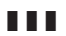

Loïc Wacquant est professeur à l'Université de Californie, Berkeley, et chercheur au Centre européen de sociologie et de science politique, Paris. Ses travaux portent sur la marginalité urbaine, la pénalité, l'incarnation et la théorie sociale. Récipiendiaire de la MacArthur Foundation Fellowship, ses ouvrages sont traduits dans une vingtaine de langues et comprennent Corps et âme. Carnets ethnographiques d'un apprenti boxeur (2000, édition anglaise augmentée 2014), Les Prisons de la misère (1999, édition augmentée et actualisée 2014), et Invitation à la sociologie réflexive (avec Pierre Bourdieu, 2014).

I www.loicwacquant.net 